\title{
Variables que inciden en el trastorno de síntomas somáticos: un modelo de regresión logística
}

\section{Variables that affect somatic symptom disorder: a logistic regression model}

\author{
Germán Benito Bernáldez-Jaimes (iD) 1, Johannes Oudhof-van-Barneveld (D)2, Érika Robles-Estrada (iD33, Alejandra del Carmen \\ Domínguez-Espinosa (iD) 4 \\ 1. Universidad Autónoma del Estado de México. Estado de México, México. Correo: mcs.gbernaldez@gmail.com - https://orcid.org/0000-0001- \\ 9538-9175 \\ 2. Universidad Autónoma del Estado de México. Estado de México, México. Correo: hansovb@hotmail.com - https://orcid.org/0000-0003-1265- \\ 3052 \\ 3. Universidad Autónoma del Estado de México. Estado de México, México. Correo: erikarobles@yahoo.com - https://orcid.org/0000-0001-7438- \\ 4500 \\ 4. Universidad Iberoamericana. Ciudad de México, México. Correo: alejandra.dominguez@ibero.mx - https://orcid.org/0000-0002-2697-8627
}

Tipología: Artículo de investigación científica y tecnológica

Para citar este artículo: Bernáldez-Jaimes GB, Oudhof-van-Barneveld J, Robles-Estrada E, Domínguez-Espinosa A. Variables que inciden en el trastorno de síntomas somáticos: un modelo de regresión logística. Duazary. 2020 octubre; 17(4): 33-45. Doi: https://doi.org/10.21676/2389783X.3598

Recibido en noviembre 01 de 2019

Aceptado en julio 28 de 2020

Publicado en línea en octubre 02 de 2020

\section{RESUMEN}

Palabras clave: síntomas somáticos; afrontamiento; personalidad.
El trastorno de síntomas somáticos (TSS) se refiere a la presencia de malestares de carácter físico acompañados de preocupaciones y conductas excesivas relacionados a la salud cuya etiología es considerada multifactorial, entre los cuales se encuentran el estrés, características personales, exposición a situaciones traumáticas y creencias irracionales. Es por ello que la presente investigación tuvo por objeto desarrollar un modelo de regresión logística con el fin de analizar la incidencia de dichos factores sobre el TSS. Se trabajó con una muestra de 201 participantes divididos en dos grupos: 111 con diagnóstico de TSS y 90 no clínicos. Se realizó un análisis de regresión logística binaria donde la prueba de ómnibus (chi cuadrado de $84,98, \mathrm{p}<0,001$ ) indica que las variables del modelo contribuyen a explicar el fenómeno. La prueba Hosmer-Lemeshow (chi cuadrado de 8,603, p=0,377) mostró además su ajuste a la realidad, con cuatro variables de riesgo, siendo las quejas gastrointestinales las de mayor proporción con un coeficiente de regresión de 1,053, seguidas de la preocupación y el estrés, introversión y pensamientos automáticos negativos, mientras que la indefensión y la desesperanza disminuyen la probabilidad del TSS. Las quejas recurrentes de carácter gastrointestinal parecen ser los factores predictores más comunes del TSS.

\section{ABSTRACT}

Keywords: somatic symptoms; coping; personality.
The Somatic Symptom Disorder (SSD) refers to the presence of physical discomforts accompanied by excessive concerns and behaviors related to health, whose etiology is presented as multifactorial, among which are stress, personal characteristics, exposure to traumatic situations and irrational beliefs. That is why this research aimed to develop a logistic regression model in order to analyze the incidence of these factors on TSS. We worked with a sample of 201 participants divided into two groups, 111 with a diagnosis of SSD and 90 non-clinical. A binary logistic regression analysis was performed, where the Omnibus test (Chi square of 84.982, $p<.001$ ) indicates that the variables of the model contribute to explain the phenomenon; additionally, the Hosmer-Lemeshow test (Chi square of 8.603, $p=.377$ ) indicates its adjustment to reality, with four risk variables. The Gastrointestinal Complaints obtained the 
highest proportion with a Regression Coefficient of 1.053, followed by Concern and Stress, Introversion and Negative Automatic Thoughts; while Helplessness and Despair decrease the probability of SSD. Recurring gastrointestinal complaints appear to be the most common predictive factors of SSD.

\section{INTRODUCCIÓN}

El trastorno de síntomas somáticos (TSS) se define como la presencia de un conjunto de malestares físicos aunados a la preocupación por la salud, pensamientos desproporcionados y un nivel elevado de ansiedad ${ }^{1}$, los cuales interfieren en el funcionamiento del individuo. Morton ${ }^{2}$ señala que pacientes que buscan atención médica general en ausencia de enfermedades físicas tienden a describir síntomas recurrentes de fatiga y dolor con ausencia de una confirmación objetiva definitiva, lo que provoca diagnósticos inadecuados.

La identificación del TSS es relativamente nueva. Investigaciones ${ }^{3}$ indican que este tipo de pacientes son complejos en múltiples niveles, por lo que requieren un tratamiento individual $y$ multidisciplinario que en la mayoría de los casos supera los procedimientos médicos y psiquiátricos convencionales. De tal forma que el diagnóstico del padecimiento denota una serie de complicaciones, tales como la falta de herramientas de evaluación y la especificidad de los factores epidemiológicos.

Se afirma que este tipo de análisis requiere a menudo de pruebas clínicas debido a la tendencia de los pacientes a reportar mayor cantidad de síntomas. En concordancia, investigaciones previas $^{5}$ señalan que los pacientes con TSS frecuentemente reportan tres o más síntomas, aunados a preocupaciones psicológicas graves que disminuyen su funcionamiento. Algunos estudios ${ }^{6}$ señalan una propensión a presentar comorbilidad con síntomas ansiosos y emocionales, combinados con malestares físicos como mareo y vértigo, factores que pueden asociarse con la cronicidad de la psicopatología. Otros reportes añaden que entre los factores etiológicos se pueden encontrar negligencia, abuso sexual, estilo de vida caótico, una historia de abuso de alcohol y otras sustancias, trastornos de la personalidad, estrés psicosocial y aspectos culturales ${ }^{7}$.

Existen diversos modelos teóricos que analizan el proceso de salud-enfermedad y señalan la relación entre las emociones, el estrés y las características de la personalidad para abordar lo somático ${ }^{8}$. En lo que se refiere al análisis de los síntomas somáticos, se ha resaltado el papel de las creencias y alteraciones perceptivas relacionadas al dolor físico, en cuanto a su exageración o preocupación excesiva por parte de los pacientes. Esto extiende la sintomatología y la relación con el signo físico, desembocando en una psicopatología somática ${ }^{9}$.

Respecto a la personalidad, se sugiere que existe una relación entre el neuroticismo y la extroversión y la aparición de síntomas somáticos, así como una asociación con el trastorno de estrés postraumático ${ }^{10}$. Asimismo, las creencias irracionales parecen desempeñar un papel preponderante en la forma en que se enfrentan las situaciones de vida; en muchos casos son factores definitorios o consecuentes de la personalidad que parecen determinar la forma en que personas con síntomas somáticos sobrellevan sus emociones.

Por otra parte, existen diversos mecanismos para afrontar las situaciones contextuales, generalmente traumáticas o percibidas como negativas. Se ha señalado una tendencia a mostrar conductas de evitación, resignación, desconexión mental y distanciamiento con la presencia de sintomatología psicopatológica de carácter somático, así como la disminución de experimentar bienestar psicológico ${ }^{11}$. Es así que la forma de percibir los estímulos parece relacionarse con la capacidad de establecer mecanismos para afrontar situaciones adversas. En concordancia, se menciona que las creencias limitantes generan experiencias de culpa, frustración, enojo y bloqueos, lo que puede desencadenar alteraciones en la salud física y emocional - paralizando la búsqueda de salidas - y resentimiento, particularmente en mujeres ${ }^{12}$. Al respecto, se advierte que el afrontamiento influye en la aparición de los síntomas psicosomáticos, en especial las estrategias de evitación, religión y autofocalización negativa, que inciden sobre todo en los síntomas de náuseas y/o problemas estomacales y desmayos ${ }^{13}$. 
De acuerdo con lo anterior, existe la necesidad de realizar estudios para determinar la presencia de factores predictores del TSS que contribuyan a realizar tratamientos psicoterapéuticos más adecuados para este tipo de pacientes ${ }^{6}$. En este sentido, se indica la pertinencia de contar con un modelo multifactorial que permita la comprensión de los trastornos somatomorfos a partir de distintas variables ${ }^{14}$. Es por ello que la presente investigación tuvo como objetivo desarrollar un modelo de regresión logística con el fin de analizar la incidencia de variables de personalidad, sucesos vitales estresantes, afrontamiento del estrés y pensamientos automáticos negativos en la presencia del TSS. Adicionalmente, se realizaron comparaciones de estas variables entre participantes sin TSS y pacientes.

\section{MATERIALES Y MÉTODOS}

\section{Tipo de investigación}

La presente investigación es de tipo explicativo y tuvo como finalidad determinar el carácter predictivo de diversas variables sobre la presencia del TSS. Además, corresponde a un estudio comparativo entre dos grupos de participantes, con diagnóstico del trastorno y sin este, con el fin de detectar las diferencias y similitudes en las variables entre ambos.

\section{Participantes}

La muestra estuvo conformada por un total de 201 participantes clasificados en dos grupos. El primero incluyó un total de 111 pacientes diagnosticados con TSS provenientes de instituciones públicas/privadas y consultorios de salud mental en el Estado de México, con una edad promedio de $24 \pm 7$ años (18 a 55 años): 43 varones y 68 féminas. En cuanto al estado civil, 69 participantes eran solteros, 37 se encontraban en matrimonio, dos eran divorciados, $y$ tres eran viudos. Respecto a escolaridad, 60 de los individuos tenían licenciatura, 33 habían hecho una carrera técnica, 11 contaban con posgrado y siete correspondían al nivel básico.

El segundo grupo estuvo conformado por 90 participantes de instituciones de educación pública y privada, con una edad promedio de $24 \pm 7$ años (18 a 55 años): 38 hombres y 52 mujeres. El estado civil para 65 de los casos fue soltero, 20 eran casados, cuatro se encontraban divorciados y uno era viudo. La escolaridad más frecuente fue de licenciatura, con 59 casos, seguida de carrera técnica (17), posgrado (10) y educación básica (4). Se utilizó un muestreo no probabilístico por conveniencia, informando a los participantes sobre el objetivo del estudio y solicitando su consentimiento informado.

\section{Instrumentos}

Los participantes con TSS fueron elegidos mediante un diagnóstico médico-psicológico, y posteriormente se aplicaron dos instrumentos para valorar la presencia de la sintomatología, los cuales fueron validados para la presente investigación. Primero se identificó la equivalencia del constructo por medir y se realizó una traducción lingüística y adaptación cultural mediante procesos de traducción y retrotraducción. Posteriormente se validaron mediante jueces la pertinencia, la claridad y la redacción de los ítems e instrucciones y se llevaron a cabo análisis factoriales exploratorios y confirmatorios.

El primer instrumento fue el Cuestionario de Experiencias de Síntomas Somáticos, creado por Herzog et $a l^{15}$, cuyo objetivo es valorar los factores psicológicos y las características interactivas pertinentes a los pacientes con TSS. Esta herramienta, validada para la presente investigación, consta de 15 reactivos con seis opciones de respuesta (nunca, muy rara vez, pocas veces, a menudo, frecuentemente y siempre), cuenta con una varianza explicada de $56,32 \%$ y está estructurada en cuatro factores: preocupación por la salud, experiencias de la enfermedad, problemas en la interacción con médicos, y consecuencias de la enfermedad. El alfa de Cronbach reportado para cada factor es de 0,83, 0,80, 0,79 y 0,80, respectivamente, para un valor total de 0,90.

El segundo instrumento empleado en la investigación fue la Escala de Síntomas Somáticos 8, creada por Gierk et $a l^{16}$. Esta herramienta unidimensional, validada para la investigación, consta de ocho reactivos en una escala tipo Likert 
con cinco opciones de respuesta (para nada, un poco, algo, bastante y mucho) y tiene un alfa de Cronbach reportado de 0,81 y una varianza total explicada de $52,67 \%$.

Para la evaluación de las variables de personalidad, pensamientos automáticos negativos, afrontamiento del estrés y sucesos vitales estresantes que se incluyeron en el modelo, se utilizaron los siguientes instrumentos:

a) Inventario Multifásico de la Personalidad de Minnesota 2 Forma Reestructurada (MMPI2 $\mathrm{RF})$ : instrumento con respuesta dicotómica (verdadero y falso) desarrollado por BenPorath y Tellegen a partir del MMPI-2, el cual ha sido validado en México por GómezMaqueo ${ }^{17}$. Está compuesto por un total de 338 reactivos estructurados en 51 escalas que conforman cuatro perfiles: personalidad psicopatológica, intereses, problemas específicos y escalas clínicas. La confiabilidad y la validez del inventario fueron establecidas mediante un estudio comparativo y de correlaciones de las puntuaciones de las escalas obtenidas del MMPI-2 y del MMPI-2 RF. Los coeficientes de confiabilidad se obtuvieron al hacer un estimado de los valores test-retest, cuyos puntajes oscilan de 0,40 a 0,90, y un alfa interno de 0,34 a 0,83 para la muestra normativa. En relación con la validez, se señala la creación de siete escalas con el objetivo de reducir las amenazas de inconsistencia sobre la prueba ${ }^{18}$.

b) Cuestionario de Pensamientos Automáticos Negativos (ATQ): validado en México por Mera et $a l^{19}$, basado en el instrumento original de Hollond y Kendall ${ }^{20}$ para la Universidad de Minnesota en Estados Unidos de América, el cual consta de 30 reactivos con una escala de respuesta tipo Likert con cinco opciones de respuesta que van desde "para nada" hasta "todo el tiempo". El cuestionario se encuentra estructurado en tres factores: 1) disgusto consigo mismo y con sus circunstancias (15 ítems); 2) sensación de culpa y juicio negativo de otros hacia su persona ( 7 ítems), y 3) sensación de ineficacia (8 ítems). Cuenta con un alfa reportado de 0,95 y una varianza explicada de $52,45 \%$.

c) Cuestionario de Afrontamiento del Estrés (CAE): es un instrumento desarrollado por Sandín y Chorot ${ }^{21}$ en España, con un alfa de Cronbach reportado de 0,79 y una varianza explicada de $49,3 \%$. Consta de siete dimensiones clasificadas en dos factores (afrontamiento racional y emocional), con una escala de respuesta de tipo Likert de cinco opciones que va desde nunca a siempre. Fue validado en México por González y Landero ${ }^{22}$, quienes desarrollaron una versión de 21 reactivos con un alfa de Cronbach de 0,78 y una varianza explicada de $40 \%$ para el factor de afrontamiento racional, y 0,71 y $60 \%$ para el afrontamiento emocional.

d) Escala de Sucesos Vitales Estresantes (SV): es un instrumento creado en España por Sandín y Chorot $^{23}$ cuyo objetivo es evaluar los sucesos estresantes en cuanto a preocupación, percepción del evento y predicción del hecho. Este presenta coeficientes alfa de Cronbach que oscilan entre 0,68 y 0,83 y una varianza explicada de $40 \%$. Consta de 60 reactivos divididos en seis dimensiones: escolar, pareja, familia, social, salud y recursos económicos. Es una escala tipo Likert con cuatro opciones de respuesta de acuerdo con el nivel de preocupación que va desde nada a muchísimo. A la par, incluye dos cuestionamientos dicotómicos en relación con la evaluación del suceso (positivo, negativo) y la aparición de este (esperado, inesperado). El instrumento cuenta con un alfa de Cronbach de 0,84 y una varianza explicada de $55 \%$ para muestras mexicanas ${ }^{24}$.

\section{Procedimiento}

Para obtener los datos se aplicaron los instrumentos, previa firma de un consentimiento informado (en forma individual para pacientes con TSS y grupal para los otros participantes). De forma posterior se codificaron los datos obtenidos y se 
procedió al análisis mediante el paquete estadístico SPSS (Statistical Package for the Social Sciences), versión 25 . También se comprobaron los supuestos de normalidad, homocedasticidad e interdependencia, donde se hallaron varianzas iguales y la no interdependencia de acuerdo con la robustez de la prueba, aunque no se aplica la normalidad para todos los grupos. Simultáneamente, se obtuvieron los datos descriptivos, se prosiguió a realizar la comparación de medias mediante la t de Student, correlaciones a través del coeficiente de Pearson y, por último, el análisis de la regresión logística binaria para conocer la incidencia de las variables predictoras sobre un variable criterio cualitativa dicotómica (Dx), para así obtener una estimación ajustada de la probabilidad de ocurrencia del TSS.

\section{Declaración sobre aspectos éticos}

La selección y la evaluación de los participantes se realizaron siguiendo los protocolos éticos de la Declaración de Helsinki y la Ley General de Salud en materia de investigación, garantizando así la colaboración voluntaria y confidencial. La presente investigación fue revisada y aprobada por el Subcomité de Enseñanza, Investigación y Ética del Centro Médico Lic. Adolfo López Mateos, con fecha del 15 de diciembre de 2017.

\section{RESULTADOS}

La comparación de las medias entre los grupos de participantes con TSS y sin trastorno se hizo mediante la $\mathrm{t}$ de Student. De este modo se obtuvieron diferencias estadísticamente significativas para todos los 20 factores de las variables de pensamientos automáticos, personalidad y afrontamiento del estrés. De estos, 19 presentan medias mayores para los pacientes con TSS, y únicamente en el caso de indefensión y desesperanza (INDEF) las puntuaciones fueron más altas para el grupo de participantes sin trastorno. Con relación al tamaño del efecto (d de Cohen), se observan efectos grandes para las variables $(d<0,80)$ CR1 y QG. Esta última refleja una diferencia de más de una desviación estándar entre los grupos, mientras que para PAC, PAl y PE se observan puntajes medios $(d<0,50)$ y, para el resto de las variables, pequeños $(d<0,20)^{25}$ (Tabla 1$)$. 
Tabla 1. Comparación de medias entre pacientes con diagnóstico de TSS y participantes sin trastorno.

\begin{tabular}{|c|c|c|c|c|c|c|c|c|}
\hline & & \multicolumn{2}{|l|}{ Sin TSS } & \multicolumn{2}{|c|}{ Con TSS } & \multirow[b]{2}{*}{$\mathbf{T}$} & \multirow[b]{2}{*}{$\mathbf{P}$} & \multirow[b]{2}{*}{$\begin{array}{l}\text { d de } \\
\text { Cohen }\end{array}$} \\
\hline & & Media & DE & Media & DE & & & \\
\hline \multirow{3}{*}{ ATQ } & PAD & 7,20 & 11,35 & 12,58 & 11,09 & $-3,38$ & 0,001 & 0,47 \\
\hline & PAC & 6,21 & 4,16 & 9,91 & 5,34 & $-5,38$ & 0,000 & 0,77 \\
\hline & PAI & 6,77 & 6,48 & 10,73 & 7,26 & $-4,04$ & 0,000 & 0,58 \\
\hline \multirow{3}{*}{ CAE } & CAEAFN & 3,08 & 2,33 & 4,04 & 2,47 & $-2,81$ & 0,006 & 0,40 \\
\hline & CAEREP & 6,17 & 1,76 & 6,86 & 2,26 & $-2,36$ & 0,019 & 0,34 \\
\hline & AFRONEMOC & 13,43 & 4,79 & 15,04 & 5,20 & $-2,25$ & 0,026 & 0,32 \\
\hline \multirow{14}{*}{ MMPI2-RF } & AEPI & 11,91 & 6,79 & 14,30 & 6,65 & $-2,50$ & 0,013 & 0,36 \\
\hline & CR1 & 7,06 & 4,49 & 10,59 & 4,07 & $-5,84$ & 0,000 & 0,82 \\
\hline & CR2 & 4,84 & 3,27 & 5,82 & 3,26 & $-2,11$ & 0,036 & 0,30 \\
\hline & CR7 & 7,68 & 4,59 & 9,14 & 5,11 & $-2,11$ & 0,036 & 0,30 \\
\hline & NEGE & 7,73 & 3,75 & 9,36 & 3,71 & $-3,08$ & 0,002 & 0,44 \\
\hline & INTR & 7,54 & 3,32 & 8,73 & 3,84 & $-2,31$ & 0,022 & 0,33 \\
\hline & MAL & 2,82 & 1,77 & 3,38 & 1,79 & $-2,20$ & 0,029 & 0,31 \\
\hline & QG & 0,69 & 0,92 & 1,95 & 1,25 & $-7,96$ & 0,000 & 1,15 \\
\hline & QDC & 1,92 & 1,72 & 2,66 & 1,51 & $-3,23$ & 0,001 & 0,46 \\
\hline & QN & 2,03 & 1,91 & 2,86 & 1,99 & $-2,97$ & 0,003 & 0,43 \\
\hline & INDEF & 1,29 & 0,94 & 1,03 & 0,89 & 2,01 & 0,045 & $-0,28$ \\
\hline & $\mathrm{PE}$ & 2,51 & 1,81 & 3,41 & 1,72 & $-3,61$ & 0,000 & 0,51 \\
\hline & ANS & 0,80 & 1,02 & 1,14 & 1,14 & $-2,24$ & 0,026 & 0,32 \\
\hline & $\mathrm{TE}$ & 3,09 & 1,92 & 3,79 & 1,98 & $-2,54$ & 0,012 & 0,36 \\
\hline
\end{tabular}

PAD: disgusto; PAC: culpa; PAI: ineficacia; CAEAFN: focalización negativa; CAEREP: reevaluación positiva; AFRONEMOC: afrontamiento emocional; AEPI: alteraciones emocionales; CR1: quejas somáticas; CR2: escasez de emociones positivas; CR7: emociones negativas disfuncionales; NEGE: neuroticismo; INTR: introversión; MAL: malestar; QG: quejas gastrointestinales; QDC: quejas de dolor de cabeza; QN: quejas neurológicas; INDEF: indefensión y desesperanza; PE: preocupación y estrés; ANS: ansiedad; TE: tendencia al enojo.

Posteriormente, se revisaron las diferencias encontradas y se eligieron las variables más representativas para el análisis de acuerdo con aquellas que resultaron ser significativas en la prueba t de Student $(p<0,05)$.

Luego se determinaron las correlaciones de Pearson entre estas variables y el diagnóstico de TSS utilizando los puntajes del Cuestionario de Experiencias de Síntomas Somáticos. Así se obtuvo una relación estadísticamente significativa positiva $(p<0,01)$, aunque de carácter bajo y moderado, para siete factores, mientras que para el resto de las variables este valor fue menor a 0,05. Asimismo, se observa que solo existe una correlación negativa, que corresponde a la variable indefensión y desesperanza (Tabla 2).
En un siguiente momento se realizó un análisis de regresión logística para determinar la probabilidad de pertenencia a los grupos mediante el método hacia atrás de Wald, tomando en cuenta el diagnóstico de TSS como variable dependiente. Con esto se obtuvo un modelo de ocho pasos que involucra las variables antes señaladas.

De acuerdo con la prueba ómnibus, el modelo presenta un chi cuadrado de 84,982 , con $5 \mathrm{gl}$ y una $p=0,001$, lo cual indica que ayuda a predecir el evento; es decir, al agregar las variables independientes, se modifica la presencia del TSS. A la par, se obtuvo un porcentaje de clasificación correcta del 77,1\% (Tabla 3), por lo que el modelo se considera aceptable. 
Tabla 2. Correlaciones entre las variables con diferencias y el diagnóstico de TSS.

\begin{tabular}{|l|l|l|l|l|l|l|l|l|l|l|l|l|l|}
\multicolumn{1}{l|}{} & Dx & ATQ & AE & AEPI & CR1 & NEGE & INTR & MAL & QG & INDEF & PE & ANS & TE \\
\hline Dx & 1 & & & & & & & & & & & & \\
\hline ATQ & $0,29^{* *}$ & 1 & & & & & & & & & & & \\
\hline AE & $0,16^{*}$ & $0,46^{* *}$ & 1 & & & & & & & & & & \\
\hline AEPI & $0,17^{*}$ & $0,41^{* *}$ & $0,17^{*}$ & 1 & & & & & & & & & \\
\hline CR1 & $0,38^{* *}$ & $0,15^{*}$ & 0,08 & $0,41^{* *}$ & 1 & & & & & & & & \\
\hline NEGE & $0,21^{* *}$ & $0,20^{* *}$ & 0,05 & $0,66^{* *}$ & $0,32^{* *}$ & 1 & & & & & & & \\
\hline INTR & $0,16^{*}$ & $0,15^{*}$ & 0,04 & $0,39^{* *}$ & $0,19^{* *}$ & 0,07 & 1 & & & & & & \\
\hline MAL & $0,15^{*}$ & $0,27^{* *}$ & 0,07 & $0,58^{* *}$ & $0,35^{* *}$ & $0,44^{* *}$ & $0,31^{* *}$ & 1 & & & & & \\
\hline QG & $0,49^{* *}$ & $0,14^{*}$ & 0,09 & 0,12 & $0,58^{* *}$ & $0,23^{* *}$ & 0,01 & $0,21^{* *}$ & 1 & & & & \\
\hline INDEF & $-0,14^{*}$ & 0,04 & 0,05 & $0,38^{* *}$ & 0,13 & $0,22^{* *}$ & $0,15^{*}$ & $0,30^{* *}$ & $-0,03$ & 1 & & & \\
\hline PE & $0,25^{* *}$ & $0,19^{* *}$ & 0,08 & $0,67^{* *}$ & $0,34^{* *}$ & $0,83^{* *}$ & 0,07 & $0,41^{* *}$ & $0,26^{* *}$ & $0,23^{* *}$ & 1 & & \\
\hline ANS & $0,16^{*}$ & $0,23^{* *}$ & $-0,01$ & $0,59^{* *}$ & $0,39^{* *}$ & $0,58^{* *}$ & 0,06 & $0,37^{* *}$ & $0,27^{* *}$ & $0,28^{* *}$ & $0,53^{* *}$ & 1 & \\
\hline TE & $0,18^{*}$ & $0,16^{*}$ & $0,17^{*}$ & $0,34^{* *}$ & $0,18^{*}$ & $0,60^{* *}$ & $-0,07$ & $0,20^{* *}$ & $0,25^{* *}$ & 0,05 & $0,35^{* *}$ & $0,30^{* *}$ & 1 \\
\hline
\end{tabular}

Dx: Cuestionario de Experiencias de Síntomas Somáticos; ATQ: pensamientos automáticos negativos; AE: afrontamiento emocional; AEPI: alteraciones emocionales; CR1: quejas somáticas; NEGE: neuroticismo; INTR: introversión; MAL: malestar; QG: quejas gastrointestinales; INDEF: indefensión y desesperanza; PE: preocupación y estrés; ANS: ansiedad; TE: tendencia al enojo. ${ }^{*} p<0,05 ;{ }^{* *} p<0,01$.

Tabla 3. Tabla de clasificación.

\begin{tabular}{|c|c|c|c|c|}
\hline \multirow[b]{2}{*}{$\begin{array}{ll}\text { N. } & \\
\text { paso } & \text { de }\end{array}$} & \multirow[b]{2}{*}{ Diagnóstico } & \multicolumn{2}{|c|}{ Diagnóstico esperado } & \multirow[b]{2}{*}{$\begin{array}{l}\text { Porcentaje } \\
\text { correcto }\end{array}$} \\
\hline & & Sin trastorno & $\begin{array}{l}\text { Trastorno de síntomas } \\
\text { somáticos }\end{array}$ & \\
\hline \multirow[t]{3}{*}{ Paso 4} & Sin trastorno & 69 & 21 & 76,7 \\
\hline & $\begin{array}{lll}\text { Trastorno de } & \text { síntomas } \\
\text { somáticos } & & \\
\end{array}$ & 25 & 86 & 77,5 \\
\hline & Porcentaje global & & & 77,1 \\
\hline
\end{tabular}

Respecto al resumen del modelo, los coeficientes de determinación presentan una proporción de variabilidad explicada de $34,5 \%$ según la $R^{2}$ de Cox y Snell y de $46,1 \%$ de acuerdo con la $R^{2}$ de Nagelkerke (Tabla 4). Esto señala que en promedio la predicción de la variable dependiente en más de un $30 \%$ por las independientes.

En relación con el ajuste del modelo, la prueba de Hosmer-Lemeshow presenta un chi cuadrado de 8,603 y una significancia de 0,377 , lo cual indica que el modelo se ajusta a la realidad. De tal manera, se acepta la hipótesis nula que señala que el modelo propuesto se ajusta a lo observado.
De acuerdo a los datos obtenidos, se mostró que las variables que predicen el incremento del riesgo de la presencia del TSS son en primer lugar las quejas gastrointestinales $(Q G)$, seguidas de preocupación y estrés (PE), introversión (INTR) y pensamientos automáticos negativos (ATQ), siendo la primera la de mayor proporción, mientras que indefensión y desesperanza (INDEF) resulta ser una variable que disminuye la probabilidad del TSS (Tabla 4). 
Tabla 4. Análisis de regresión logística binaria.

\begin{tabular}{|l|l|l|l|l|l|l|l|l|}
\hline Variable & B & ET & Wald & \multirow{2}{*}{$\mathbf{g l}$} & $\mathbf{p}(\mathbf{0 , 0 5})$ & \multirow{2}{*}{ Exp(B) } & \multicolumn{2}{l|}{ IC 95\% para EXP(B) } \\
\hline INTR & 0,122 & 0,053 & 5,352 & 1 & 0,021 & 1,130 & 1,019 & Inferior \\
\hline INDEF & $-0,580$ & 0,204 & 8,072 & 1 & 0,004 & 0,560 & 0,375 & 1,254 \\
\hline PE & 0,230 & 0,108 & 4,540 & 1 & 0,033 & 1,259 & 1,019 & 1,835 \\
\hline ATQ & 0,024 & 0,008 & 8,087 & 1 & 0,004 & 1,024 & 1,007 & 1,041 \\
\hline QG & 1,053 & 0,188 & 31,235 & 1 & 0,000 & 2,865 & 1,981 & 4,145 \\
\hline Constante & $-2,720$ & 0,619 & 19,311 & 1 & 0,000 & 0,066 & & \\
\hline
\end{tabular}

INTR: introversión; INDEF: indefensión y desesperanza; PE: preocupación y estrés; ATQ: pensamientos automáticos negativos; QG: quejas gastrointestinales.

\section{DISCUSIÓN}

La presencia de los síntomas somáticos ha sido vinculada a una serie de factores, entre los cuales se encuentran la personalidad, la percepción, la cognición, acontecimientos vitales, estrés, afrontamiento y la conceptualización de la enfermedad ${ }^{14}$, por lo que la identificación de las diferencias entre una muestra clínica y personas sin trastorno permite identificar aquellos factores primordiales para el análisis. De acuerdo a los resultados del presente estudio, las quejas gastrointestinales y los pensamientos automáticos negativos son los factores estadísticamente significativos con mayor presencia en los pacientes con TSS, lo cual es concordante con un estudio que indica que los pacientes con somatización presentan mayores dilemas cognitivos ${ }^{26}$.

El análisis correlacional revela una relación de baja a moderada entre las quejas somáticas, las gastrointestinales y los pensamientos automáticos con la manifestación de SS, lo cual parece indicar que el conjunto de preocupaciones relacionadas con la salud física son lo que origina la tendencia a desarrollar y aumentar el malestar percibido. Al respecto, se explica la existencia de una relación directa entre intensidad sintomática y la presencia de ansiedad ${ }^{27}$.

EI TSS involucra la presencia de una serie de malestares de carácter físico, los cuales denotan un conjunto de preocupaciones relacionadas a la salud, así como conductas destinadas a la comprobación del padecimiento con ausencia de una explicación médica. Entre los principales síntomas se ha reportado una evolución cíclica con molestias reumáticas, crisis asmáticas, molestias gástricas y dermatosis, las cuales se relacionan con la presencia de trastornos de afectividad ${ }^{28}$. En concordancia, los datos hallados en esta investigación señalan las quejas gastrointestinales como principal factor predictor, tal como se sugiere al afirmar que el dolor de estómago, el dolor de espalda, frecuentes diarreas, fuertes palpitaciones y excesivo cansancio o leve capacidad para realizar actividades son los predictores para el diagnóstico de síntomas somáticos ${ }^{29}$.

Asimismo, existe información que indica que no es necesario que los síntomas estén presentes, sino que más bien basta que estos sean narrados por el individuo. Se ha encontrado que los sistemas orgánicos más comunes son: pseudoneurológico, gastrointestinal, sistema reproductor femenino, psicosexual, dolor y cardiopulmonar ${ }^{30}$. De acuerdo a esto, los resultados del modelo de regresión concuerdan en cuanto a la predicción diagnóstica, denotando que son las quejas recurrentes a nivel gástrico los aspectos primordiales en el proceso de identificación del TSS.

La presencia de síntomas de preocupación y estrés como factores predictores representa la tendencia de los participantes a desarrollar situaciones que determinan su incapacidad directa para poner en marcha mecanismos que les hagan frente a las problemáticas que experimentan. De tal forma se desencadenan reacciones a nivel fisiológico, las 
cuales pueden ser consideradas como estímulos tensionales que provocan reacciones somáticas ${ }^{31}$. Además, se reconoce un alto grado de preocupaciones en pacientes con SS que van acompañadas de la presencia de pensamientos catastróficos $^{32}$. Asimismo, se afirma que el contenido referente a las preocupaciones psicológicas asociado a los trastornos somatomorfos y SS se relaciona con la experimentación de situaciones traumáticas y las creencias irracionales ${ }^{5}$.

En cuanto a las variables de personalidad, la introversión parece ser uno de los factores predictivos asociados al TSS, lo que indica, de acuerdo con la interpretación del MMPI2-RF, la pérdida de experiencias emocionales positivas y la evitación de situaciones e interacciones sociales ${ }^{17}$. Esto implica que no solo existe la tendencia al aislamiento del individuo a nivel social, sino también a nivel emocional, lo que se manifiesta en la incapacidad para analizar la existencia de alternativas ante las situaciones que se presentan. En este sentido, se presenta una relación entre el neuroticismo e introversión y los trastornos del dolor, mayoritariamente en la fibromialgia ${ }^{33}$.

En cuanto a los aspectos intrapersonales, los resultados del análisis contradicen ciertos hallazgos señalados anteriormente ${ }^{11,13}$ donde se sugiere la tendencia evasiva y negativa de los pacientes ante las situaciones catastróficas, pues en el presente estudio se observa, de hecho, una menor percepción de indefensión y desesperanza como factor predictivo. Esto permite inferir la propensión a desarrollar una constricción emocional como mecanismo de afrontamiento ante las situaciones descritas como traumáticas o desestabilizadoras, en lugar de tendencias evitativas, lo que da cuenta de una percepción con capacidad para superar las dificultades en busca del desarrollo de la autotrascendencia ${ }^{34}$.

Pese a la presencia del manejo perceptual de las condiciones traumáticas mediante la tendencia a desplazar el afecto a aspectos positivos, la presencia de los pensamientos automáticos de culpa denota la incapacidad de los pacientes con TSS para modular los afectos. Asimismo, la inclinación al autorreproche y la percepción de sentirse juzgado por otras personas pueden indicar la búsqueda de estrategias alternativas para someter la experimentación de las experiencias emocionales, de tal forma que se presenta el desarrollo de creencias de desamparo, desesperanza y depresión ${ }^{35,15}$.

En tal sentido, la falta de establecimiento de lazos sociales y afectos con otros, aunada al desinterés y la incapacidad para mantener decisiones, parece generar en los sujetos el desarrollo de preocupaciones. Es así como el aislamiento se estructura como un mecanismo de afrontamiento ante las problemáticas, aspecto que supone la incapacidad para experimentar frustración y angustia, síntomas característicos de la somatización ${ }^{34}$.

En conclusión, las quejas gastrointestinales recurrentes resaltan las diferencias entre los grupos, seguidas de las somáticas, la culpa, indefensión y la presencia de preocupación y estrés. A la par, se observa que existen relaciones positivas moderadas bajas entre los problemas estomacales, las quejas y los pensamientos negativos y el TSS, por lo que se afirma que se presenta una consistencia de los hallazgos obtenidos en relación con los SS.

Las quejas gastrointestinales parecen ser los factores predictores más comunes del TSS, por lo que constituyen uno de los mejores criterios para el diagnóstico, tomando la referencia de tres síntomas - más ${ }^{5}$. A su vez se establece la tendencia a experimentar una restricción de las experiencias emocionales, lo cual implica una pérdida en la capacidad para desarrollar afectos positivos con los otros, de tal forma que el desapego y la pasividad parecen ser mecanismos para evitar la constricción de las reacciones afectivas. Es así que las personas con TSS, ante las situaciones de carácter estresante, suelen desarrollar una serie de mecanismos de afrontamiento orientados a la trascendencia (superación del problema) mediante el despliegue de estrategias que les permiten asumir los aspectos negativos vividos como experiencias de crecimiento, tendiendo a restringir la presencia de emociones que consideran dañinas. Adicionalmente, suelen elaborar procesos cognitivos de razonamiento 
perjudiciales, en tanto que tienden a percibir críticas hacia su persona por parte de los otros, lo que

La presente investigación tiene como limitación que los resultados no son generalizables a la población en general. Sin embargo, permite identificar factores para la predicción del TSS y vislumbrar la necesidad de establecer mecanismos adecuados de diagnóstico para afinar los análisis predictivos. EI estudio además sugiere que la represión emocional y la evasión de la situación traumática son aspectos primordiales en la sintomatología. Finalmente, si bien los resultados apoyan la presencia de factores de aislamiento y evitación afectiva, pesimismo, anhedonia, síntomas depresivos, así como la falta de interacción con otros, por otra parte, cabe añadir que la tendencia a la búsqueda de cambio, pese a la percepción negativa, disminuye la sintomatología. Por lo tanto, es necesario hacer una revisión sobre los comportamientos sociales de los pacientes con TSS y los mecanismos de compromiso emocional en futuros estudios.

\section{AGRADECIMIENTOS}

Se agradece especialmente al Centro Médico Lic. Adolfo López Mateos de la ciudad de Toluca, del Estado de México, hospital de tercer nivel de alta especialidad, y a su honorable comité de ética por su colaboración y apoyo.

\section{DECLARACIÓN SOBRE CONFLICTOS DE INTERESES}

Los autores declaran que no existe conflicto de intereses.

\section{CONTRIBUCIÓN DE LOS AUTORES}

Primer autor: trabajo de campo, redacción, diseño metodológico y análisis estadístico.

Segundo autor: trabajo de campo, redacción, diseño metodológico y análisis estadístico.

Tercer autor: diseño metodológico y análisis estadístico.

Cuarto autor: diseño metodológico y análisis estadístico. proyecta deterioro en la autoconfianza y así lleva al desarrollo de los síntomas somáticos.

\section{REFERENCIAS BIBLIOGRÁFICAS}

1. Asociación Americana de Psiquiatría. Guía de consulta de los criterios diagnósticos del DSM-5. Washington-Londres: American Psychiatric Publishing; 2014.

2. Morton T. Somatic Symptom Disorders without known physical causes: One disease with many names? AJM. 2015 [citado 7 abr 2018]; 128(10): 1054-1058.

Doi: http://dx.doi.org/10.1016/j.amjmed.2015.04.041

3. Van Eck, J, De Vroege L, Van Manen A, Van der Thiel E, Timmermans A, Pouwer $\mathrm{F}$, et al. Case complexity in outpatients in a centre of excellence for somatic symptom disorder -a cross-sectional study-. J. Psychosom. Res. 2016 [citado 7 abr 2018]; 85: 85-85. Doi: http://dx.doi.org/10.1016/j.jpsychores.2016.03.2

4. Carson A, Stone J, Hansen C, Duncan R, Cavanagh $J$, Matthews K, et al. Somatic symptom count scores do not identify patients with symptoms unexplained by disease: a prospective cohort study of neurology outpatients. J. Neurol. Neurosurg. Psychiatry. 2015 [citado 9 abr 2018]; 86: 295-301. Doi: http://dx.doi.org/10.1136/jnnp-2014-308234

5. Van Geelen S, Rydelius P, Hagquist C. Somatic symptoms and psychological concerns in a general adolescent population: Exploring the relevance of DSM-5 somatic symptom disorder. J. Psychosom. Res. 2015 [citado 9 abr 2018]; 79(4): 251-258. Doi: http://dx.doi.org/10.1016/j.jpsychores.2015.07.01

6. Limburg K, Sattel H, Dinkel A, Radziej K, BeckerBense S, Lahmannb C. Course and predictors of DSM-5 somatic symptom disorder in patients with vertigo and dizziness symptoms - A longitudinal study. Compr. Psychiatry. 2017 [citado 9 abr 2018]; 77: 1-11. Doi: http://dx.doi.org/10.1016/j.comppsych.2017.05.00 3 
7. Kurlansik S, Maffei M. Somatic Symptom Disorder. AAFP. 2015 [citado 15 abr 2018]; 93(1):49-54. PMID: 26760840.

8. Veloz J, Lorenzo A. La somatización: desde una mirada psicosocial a los estudios de la personalidad. Revista Electrónica de Psicología Iztacala [revista en la Internet]. 2017 [citado 2018 Abr 15]; 19(4): 14431465.

Disponible

en: https://www.medigraphic.com/cgi-

bin/new/resumen.cgi?IDARTICULO=72487

9. Angenendt J, Harter M. Somatoform Disorders. Munich: Elsevier; 2015.

10. Breslau N, Davis G, Andresky P. Risk factors for Post-traumatic Stress Disorder related with traumatic events: a prospective analysis. Am. J. Psychiatry. 1995 [citado 10 abr 2018]; 152: 529-35. Doi: http://dx.doi.org/10.1176/ajp.152.4.529

11. Bruno F, De Rosa S, Stover J, De la Iglesia G. Afrontamiento al estrés, apoyo social percibido y sintomatología psicopatológica: estudio preliminar en Buenos Aires. IV Congreso Internacional de Investigación y Práctica Profesional en Psicología. XIX Jornadas de Investigación VIII Encuentro de Investigadores en Psicología del Mercosur. Facultad de Psicología - Universidad de Buenos Aires, Buenos Aires; 2012. [Citado 25 jul 2017]. Disponible en: https://www.aacademica.org/000-072/961.pdf

12. Dorantes M. Desarrollo profesional versus atención de la familia: dilema al que se enfrentan las profesionistas. Alternativas en Psicología. 2015 [citado 25 jun 2017]; 18: 74-86. Disponible en: https://www.alternativas.me/attachments/article/ 92/6\%20-

\%20Desarrollo\%20profesional\%20versus\%20atenci \%C3\%B3n\%20de\%20la\%20familia.pdf

13. Pancho L, Procel C. Afrontamiento del estrés y síntomas psicosomáticos en pacientes atendidos en hospital alternativo Andino de Riobamba, período agosto 2016-enero 2017 (Tesis de Licenciatura). Recuperada de la Red de Repositorios de Acceso Abierto del Ecuador. [Citado 25 jul 2017]. Disponible en: http://dspace.unach.edu.ec/bitstream/51000/3978 /1/UNACH-EC-FCS-PSC-CLIN-2017-0025.pdf

14. Kapfhammer H. Somatization-somatoform disorders-etiopathological models. Fortschritte der Neurologie-Psychiatrie. 2001 [citado 7 abr 2018]; 69(2): 58-77. Doi: http://dx.doi.org/10.1055/s2001-11172

15. Herzog A, Voigt K, Meyer B, Wollburg E, Weinmann N, Langs $G$, et al. Psychological and interactional characteristics of patients with somatoform disorders: Validation of the Somatic Symptoms Experiences Questionnaire (SSEQ) in a clinical psychosomatic population. J. Psychosom. Res. 2015 [citado 13 abr 2018]; 78(6): 553-562. Doi: http://dx.doi.org/10.1016/j.jpsychores.2015.03.004

16. Gierk B, Kohlmann S, Kroenke K, Spangenberg L, Zenger $\mathrm{M}$, Brähler $\mathrm{E}$, et al. The Somatic Symptom Scale-8 (SSS-8): A Brief Measure of Somatic Symptom Burden. JAMA Internal Medicine. 2014 [citado 10 abr 2018]; 174(3): 399-407. Doi: http://dx.doi.org/10.1001/jamainternmed.2013.12 179

17. Gómez-Maqueo E. Manual de aplicación, calificación e interpretación: Inventario multifásico de la personalidad de Minnesota-2 forma reestructurada. Ciudad de México: Manual Moderno; 2015.

18. Arenas P, Martínez L. Inventario multifásico de la personalidad de Minnesota-2 Forma reestructurada: Manual técnico. México: Manual Moderno; 2015.

19. Mera A, Hernández M, Gómez J, Ramírez N, Mata M. Características psicométricas del cuestionario de pensamientos automáticos negativos (ATQ-30) en población mexicana. Journal of Behaviour, Health y Social Issues. 2012 [citado 13 may 2018]; 3(2): 61-75. Doi: http://dx.doi.org/10.5460/jbhsi.v3.2.29923

20. Hollon SD, Kendall PC. Cognitive self-statements in depression: development of an automatic thoughts questionnaire. Cognitive Therapy and 
Research. 1980 [citado 20 jul 2017]; 4(4): 383-395. Doi: https://doi.org/10.1007/BF01178214

21. Sandín B, Chorot P. Cuestionario de afrontamiento del estrés (CAE): desarrollo y validación preliminar. Revista de Psicopatología y Psicología Clínica. 2003 [citado 2018 May 13]; 8(1): 39-54.

Doi:

http://dx.doi.org/10.5944/rppc.vol.8.num.1.2003.3 941

22. González M, Landero R. Cuestionario de afrontamiento del estrés (CAE): Validación en una muestra mexicana. Revista de Psicopatología y Psicología Clínica. 2007 [citado 17 may 2018]; 12(2): 189-198.

Doi: http://dx.doi.org/10.5944/rppc.vol.12.num.3.2007. 4044

23. Sandín B, Chorot P. Evaluación de los sucesos vitales. En B. Sandín (Ed.). El estrés psicosocial: Conceptos y consecuencias clínicas. Segunda edición. Madrid: Klinik; 2008. p. 65-83.

24. Veytia M, González-Arratia N, Andrade $P$, Oudhof H. Depresión en adolescentes: El papel de los sucesos vitales estresantes. Salud Mental. 2012 [citado 2017 May 15]; 35(1): 37-43. Disponible en: http://www.scielo.org.mx/scielo.php?script=sci_art text\&pid=S0185-33252012000100006

25. Cohen J. Statistical power analysis for the behavioral science. Nueva York: LEA; 1988.

26. Freixas G, Cipriano D, Varlotta N. Somatización y conflictos cognitivos: estudio exploratorio con una muestra clínica. Revista Argentina de Clínica Psicológica. 2007 [citado 2017 May 15]; 16(3): 97$203 . \quad$ Disponible en: https://www.redalyc.org/pdf/2819/281921790001. pdf

27. González M, Landero R, García-Campayo J. Relación entre la depresión, la ansiedad y los síntomas psicosomáticos en una muestra de estudiantes universitarios del norte de México. Revista Panamericana de Salud Pública. 2009 [citado 2017 Jun 16]; 25(2): 141-5. Disponible en: http://iris.paho.org/xmlui/handle/123456789/987
28. Chávez K. Depresión enmascarada, ¿somatización o depresión? Psicología, Psicoanálisis y Conexiones. 2013 [citado 2017 Jun 16]; 5(8): 1-8. Disponible en: https://www.google.com.mx/url?esrc=s\&frm=1\&q $=\& r c t=j \& s a=U \& u r l=h t t p s: / / a p r e n d e e n l i n e a . u d e a . e$ du.co/revistas/index.php/Psyconex/article/downlo ad/20977/17525\&ved=2ahUKEwj0uJvnzsnIAhULLa OKHdMHCJAQFjADegQIChAB\&usg=AOVVaw2_XBLX CMeX-z0a1okVW2EL

29. Gili M, Bauzá N, Vives M, Moreno S, Magallón R, Roca M. Validación de la versión española de la SOMS-2 (screening for somatoform symptoms-2) para la evaluación de síntomas somáticos en atención primaria. Atención Primaria. 2015; 47(5): 273-278. Doi: http://dx.doi.org/10.1016/j.aprim.2014.07.001

30. López J, Belloch A. La somatización como síntoma y como síndrome: una revisión del trastorno de somatización. Revista de Psicopatología y Psicología Clínica. 2002; 7(2): 73-93. Doi:

http://dx.doi.org/10.5944/rppc.vol.7.num.2.2002.3 923

31. Caballero L, Caballero F. Trastornos somatomorfos y síndromes somáticos funcionales en atención primaria. Monografía: Salud mental en la atención primaria. JANO extra. 2008 [citado 15 may 2018]; 1714: 15-29. Disponible en: https://dialnet.unirioja.es/servlet/articulo?codigo= 2752014

32. Vijay KG, Avasthi A, Grover S. A study of worry and functional somatic symptoms in generalized anxiety disorder. Asian Journal of Psychiatry. 2014 [citado 21 may 2018]; 11: 50-52. Doi: http://dx.doi.org/10.1016/j.ajp.2014.05.011

33. Fernández A, López M. Estudio de la asociación entre la fibromialgia y el tipo de personalidad (Tesis Doctoral). [Citado 8 sep 2017]. Disponible en: https://dialnet.unirioja.es/servlet/tesis?codigo $=128$ 874

34. Espinosa J, Contreras F, Esguerra G. Afrontamiento al estrés y modelo psicológico de la 
personalidad en estudiantes universitarios. Diversitas. 2009 [citado 2018 Sep 8]; 5(1): 87-95.

Disponible

en:

https://www.redalyc.org/pdf/679/67916259008.pd

f

35. Landero R, González M. Variables psicosociales como predictoras de la salud en mujeres. Revista psicosocial en México. 2004 [citado 5 sep 2017]; 19(2): 255-264. Doi:

http://dx.doi.org/10.1174/0213474041960504 